\title{
The $v$-Ball campaign at ALTO: Study for neural network based trigger for fission process
}

\author{
Matthieu Lebois ${ }^{1,2, *}$, Nikola Jovančević ${ }^{3}$, Damien Thisse ${ }^{1}$, Jonathan Wilson ${ }^{1}$, \\ Rhiann Canavan ${ }^{4,5}$, Mathias Rudigier ${ }^{4}$, and the N-SI-109 experimental team \\ ${ }^{1}$ Université Paris-Saclay, CNRS/IN2P3, IJCLab, 91405 Orsay, France \\ ${ }^{2}$ Institut Universitaire de France, 1 Rue Descartes, 75005 Paris, France \\ ${ }^{3}$ Department of Physics, Faculty of Science, University of Novi Sad, Trg Dositeja Obradovica 3, 21000 \\ Novi Sad, Serbia \\ ${ }^{4}$ Department of Physics, University of Surrey, Guildford, GU2 7XH, UK \\ ${ }^{5}$ National Physical Laboratory, Teddington, UK
}

\begin{abstract}
A $\gamma$-spectroscopy campaign named " $v$-Ball" was perfomed at the ALTO facility. A large fraction of the beam time was dedicated to the fast neutron induced fission of two fissioning systems: ${ }^{232} \mathrm{Th}$ and ${ }^{238} \mathrm{U}$. During the data analysis, it was noticed that the high activity of the ${ }^{\text {nat }}$ Th was heavily contaminating any coincidence matrices (or cubes) built. This caused the identification of weakly produced fission fragments identification to be almost impossible. It was decided to explore the opportunity opened by new analysis methods based on neural networks algorithms. In this paper, the methods to build an adequate neural network and the results obtained for fission event reconstruction are presented.
\end{abstract}

\section{Introduction}

In 2017-2018, the ALTO facility of the "Laboratoire de Physique des 2 infinis Irène JoliotCurie" (former "Institut de Physique Nucléaire d'Orsay") hosted an experimental campaign using the $\gamma$ spectrometer named: $v$-ball. Generally, $\gamma$-ray spectroscopy is a tool used in nuclear physics research, which has permitted the gathering of great quantities of information about excited nuclear states. Over the past decade, highly detailed coincidence spectroscopy has been performed over a wide range of atomic nuclei, allowing detection of $\gamma$ transitions as weak as $10^{-5}[1-4]$ of the total reaction cross section. Information on spins and parities of these excited states can also be deduced with pure germanium detector arrays using measurement techniques such as $\gamma$-ray angular distributions, angular correlations and polarisations. Nowadays, development of fast-scintillator such as $\mathrm{LaBr}_{3}$ allows the access to lifetime information in the 10 ps to $10 \mathrm{~ns}$ range, which is complementary to germanium based lifetime measurements techniques (over a 20 ns range) or plungers based measurement - to get lifetimes in the 1-100 ps range [5, 6]. In a similar way as the ROSPHERE array [7], the $v$-Ballspectrometer has been implemented at the ALTO facility.

\footnotetext{
*e-mail: lebois@ijclab.in2p3.fr
} 


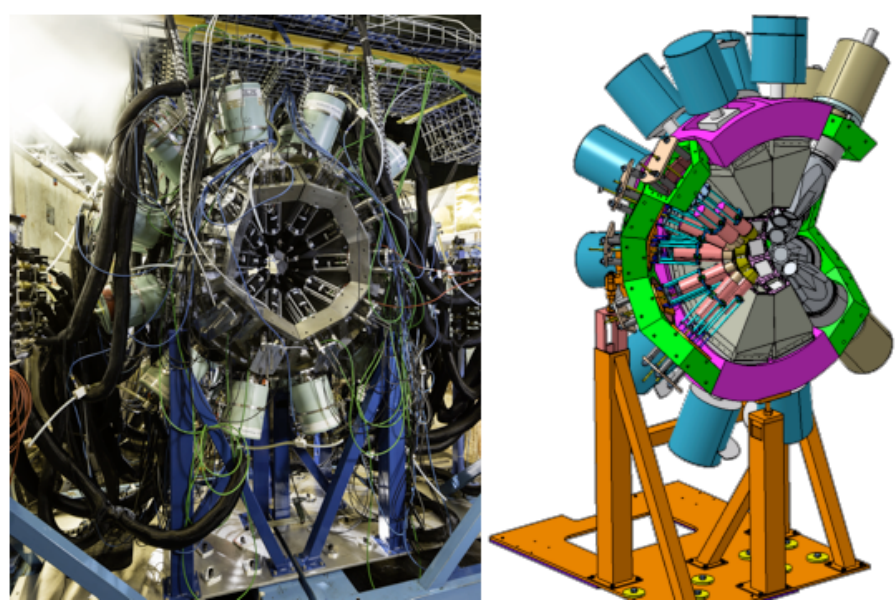

Figure 1. Photograph of the $v$-Ballarray installed in the experimental area of the ALTO facility (left panel) and a technical drawing of one $v$-Ballhemisphere (right panel).

\section{Presentation of the $v$-Ballspectrometer \& the $v$-Ballcampaign}

The $v$-Ballarray combines 24 Ge clovers from the GAMMAPOOL [8], 10 co-axial high purity germanium (HPGe) detectors and $20 \mathrm{LaBr}_{3}$ or 34 PARIS phoswich detectors [9-11]. Figure 1 shows a photo of the array (on the left). On the right a technical drawing shows the three types of detector: HPGe clover detectors and their associated Compton shield (the two central rings), co-axial HPGe detectors (right ring) and $\mathrm{LaBr}_{3}$ in the left ring.

To optimize $v$-Ballperformances, a special attention has been brought to:

- Compton rejection via the use of Compton shields. In addition, heavy metal collimators on the BGO detectors were removed to obtain an energy measurement for the event-by-event calorimetry of the nuclear reactions studied.

- Inclusion of $\mathrm{LaBr}_{3}$ detectors to facilitate measurements of excited nuclear states lifetimes in the $10 \mathrm{ps}-10 \mathrm{~ns}$ range.

- Use of a fully digital DAQ system to allow processing of very high count rates, to minimize the dead time, optimize the time resolution of all the different detector types, and to make the signal processing simpler. Such a system can be run in either triggerless or triggered mode. Triggerless mode presents the advantage of a total freedom in the event reconstruction off-line.

Since the spectrometer is intended to be used with neutron beams no detectors are placed at forward angles ( $<20$ degrees) to allow neutrons to escape the array without touching a detector - and potentially damage it. $\mathrm{The}^{\mathrm{LaBr}} 3$ detectors are preferentially placed as two forward rings since they are much more resilient to scattered neutrons. A brief description of the $v$-Balldetectors will be given in the following. More details can be found in [12].

\subsubsection{The high purity germanium detectors}

High purity germanium detectors were of two types: coaxial germanium crystals and clover detectors. 
Ten coaxial germanium detectors, named "Phase I", were placed in a ring at backward angles $\left(133.5^{\circ}\right.$ with respect to the beam axis) and $16 \mathrm{~cm}$ from the center of the sphere to the BGO shield entrance window. That sets the crystal at $18 \mathrm{~cm}$ from the center. They were rented to the FRENCH/UK loan pool [13] for the duration of the campaign.

The clover germanium detectors were borrowed from the GAMMAPOOL [8] for the duration of the campaign and are placed in two rings of twelve detectors each around $90^{\circ}$ $\left(75.5^{\circ}\right.$ and $104.5^{\circ}$ with respect to the beam axis) to take advantage of their granularity in case of Doppler corrections. The distance from the BGO shield entry to the center was $17 \mathrm{~cm}$ which places the crystals at $24 \mathrm{~cm}$ from the center. Signals from germanium crystals and BGO crystals were directly digitized without preamplification. Post treatment of the data set will allow the use of the BGO signal for calorimetry purposes at the same time as for Compton rejection. A total photopeak efficiency of $4 \%$ and an energy resolution of $2.8 \mathrm{keV}$ was measured at $1.33 \mathrm{MeV}$.

\subsubsection{The $\mathrm{LaBr}_{3}:$ Ce detectors}

The fast-timing part of the array was constituted of twenty $\mathrm{LaBr}_{3}: \mathrm{Ce}$ crystals. These detectors were provided by the FATIMA [14] and UKNDN [15] collaborations. The remaining ten percent of available solid angle in the sphere permitted the packing of 20 of these detectors in two rings of ten detectors each. The first ring was at $46.5^{\circ}$ with respect to the beam axis and $11 \mathrm{~cm}$ away from the sphere center. The second ring was at $34^{\circ}$ with respect to the beam axis and $15 \mathrm{~cm}$ away from the sphere center. When coupled to the LICORNE directional neutrons source, the $v$-BallLaBr 3 second ring had to be moved further away from the center $(\sim 25 \mathrm{~cm})$ in order to be outside the neutron cone. The reference layout of the array is presented in Fig. 1. A technical drawing from the IPNO design office of one hemisphere is presented in the right panel. Two rings of $\mathrm{LaBr}_{3}$, two rings of clovers embedded in their BGO shields and a last ring of Phase I detectors in their shields are visible from left to right. The left panel shows a photograph of the array at the beginning of the experimental campaign before the mounting of the reaction chamber.

A separate configuration involving the coupling of $v$-Ballwith four clusters of the PARIS [9-11] array was also performed. For this setup all the $\mathrm{LaBr}_{3}$ detectors were replaced by 34 PARIS phoswitch detectors almost completely covering the available solid angle in the forward direction $(\sim 30 \%)$.

Globally, an energy resolution of $2.7 \%$ and an photopeak efficiency up to $1.5 \%$ (depending on the crystals positions) was measured at $662.7 \mathrm{keV}$.

\subsection{The data acquisition system: FASTER}

All the pre-amplifier or anode signals are sent to a fully digital acquisition system called FASTER (Fast Acquisition System for nuclEar Research) [16] developed at the Laboratoire de Physique Corpusculaire in Caen, France. It is used to measure relevant observables (deposited energy and time of each hit) extracted online from the traces. FASTER has been built to be modular and fully integrated to satisfy the needs of any type of experiment in nuclear physics, particularly those, which combine detector types with significantly different characteristics. FASTER is capable of on-line processing of traces, to sort them and group them into "events" (depending on each scientific goal) through a software event builder module, no matter what is the type of detector. At the same time, data can be visualized using the versatile ROOT Histogram Builder (RHB) software. Based on ROOT [17], it allows the implementation of online energy, time-of-flight (TOF) spectra or any kind of 1D-2D user-defined histograms. All the signals of the $v$-Ballarray are sent to 184 (200 with the PARIS setup) 
independent electronics channels. These are distributed over 34 motherboards in three $\mu$ TCA crates. The crates are synchronized using an external logic signal (called "t0") distributed by an $\mathrm{MCH}$ card located in a fourth crate. The maximum count rates, obtained during the campaign, in the individual detectors are: $10 \mathrm{kHz}$ for the HPGe, $15 \mathrm{kHz}$ for the $\mathrm{LaBr}_{3}$ and $40 \mathrm{kHz}$ for the BGO crystals without having any dead time, or showing a degradation of energy resolution.

\subsection{The $v$-Ballcampaign}

The $v$-Ballspectrometer was operational over a one year period from November 2017 to June 2018. Over 150 researchers participated in the experimental campaign and approximately 3200 hours of beam time were provided by the ALTO facility. A detailed description of the campaign progress is given in [18]. Eight experiments were performed, a short description of some of them is given in [19-21]. The variety of physics cases necessitated several transformations of the geometry during the course of the campaign.

After construction a commissioning experiment was performed using an ionisation chamber with a Frisch grid containing a ${ }^{252} \mathrm{Cf}$ spontaneous fission source of $3 \mathrm{kBq}$ on a lead backing at the central cathode to stop one of the fission fragments. The chamber of $15 \mathrm{~cm}$ diameter was placed in the center of the array and the $v$-Balldata acquisition triggered with the chamber cathode signal. The test was performed to validate the DAQ system at a low count rate with fission events of an average gamma multiplicity around 8. Data were acquired for around 50 hours.

Subsequently, a first set of experiments $[19,20]$ based on heavy-ion induced fusionevaporation reactions were performed with all $v$-Balldetectors placed in the most compact geometry possible - identical to the previously simulated GEANT4 setup. The trigger condition was multiplicity of two $\gamma$ rays detected in any $\mathrm{LaBr}_{3}$ module (or one Ge module and one $\mathrm{LaBr}_{3}$ ) within $2 \mu \mathrm{s}$. Clover modules had a local trigger on the card, to provide a multiplicity of one, even in the event of more than one clover crystal firing.

Later experiments required a change in geometry to couple the array with the LICORNE neutron source [21]. The neutron source produces naturally directional neutrons in a cone that depends on the primary beam energy. It is important to remind that the neutron beam can be pulsed with a pulse width of $2 \mathrm{~ns}$ and a period of $400 \mathrm{~ns}$.

The presence of the LICORNE chamber and the heavy metal collimator, that shield the gas cell, causes a partial shadowing effect on the backward ring of coaxial germanium detectors, thus slightly lowering the total efficiency of the array. A photograph of the nuball/LICORNE coupling is presented on the right of Fig. 2. The black detectors (conical $\mathrm{LaBr}_{3}$ crystals) were retracted to a distance of $10 \mathrm{~cm}$ and the grey detectors (cylindrical $\mathrm{LaBr}_{3}$ crystals) were retracted to a distance of $15 \mathrm{~cm}$ in order to make sure the source neutrons, produced in a cone of around 20 degrees opening angle, can escape the spectrometer. The experiments using a neutron beam to study the nuclear structure of very neutron-rich fission fragments and the fission mechanism itself were performed over seven weeks of beam time. The DAQ was ran in triggerless mode, and due to the very high count rates and network bandwidth limitations, data were sent to disk at maximum rates of around $70 \mathrm{MB} / \mathrm{second}$, or 3 TB per day. The primary beam intensity was set to obtain counting rates in the individual crystals of around $10 \mathrm{kHz}$ in the HPGe, $40 \mathrm{kHz}$ in the BGO and $15 \mathrm{kHz}$ in the $\mathrm{LaBr}_{3}$. The total amount of data collected during the entire $v$-Ballcampaign was very large $(\sim 0.2 \mathrm{~PB})$, a significant proportion of which was transferred to the IN2P3 data-center [22].

The last experiment of the campaign replaced all the FATIMA $\mathrm{LaBr}_{3}$ with PARIS phoswitches. A photograph of the coupling is presented in the right panel of Fig. 2. The 34 phoswitches were placed in a cylindrical cluster arrangement at a distance of $20 \mathrm{~cm}$ from 




Figure 2. The left panel shows a photograph of the experimental setup of $v$-Ballcoupled to the PARIS array. The right panel shows the $v$-Ballarray coupled to the LICORNE neutron production chamber with a thick target of natural thorium placed at the center of the array.

the center of the array to maximize solid angle coverage. This addition provided a significant increase in the total efficiency at energies greater than $4 \mathrm{MeV}$. A further 14 FASTER CARAS channels were added to the system, making a total of 200 independent digital channels. This experiment was also run in triggerless mode to allow complete offline characterization of the cross-talk/scattering of $\gamma$-rays in the PARIS clusters.

\section{Fission studies with $v$-Ball}

As mentioned in the previous section, a part of the beam time of the $v$-Ballcampaign has been dedicated to the coupling with the LICORNE neutron source. It included three experimental projects on fission process studies: one dedicated to the search of fission isomer (experiment labeled: N-SI-103) and spectroscopy in the second potential well, the other two dedicated to study of fission fragments produced by the fast neutron induced fission of ${ }_{92}^{238} \mathrm{U}$ and ${ }_{90}^{232} \mathrm{Th}$ (experiment labeled: N-SI-109).

\subsection{Description of fission experiment with $v$-Ball}

Experiment N-SI-103 used a cylindric aluminum tube in which five pellets of depleted uranium (isotopic purity of $99.7914 \%$ ) were placed. The tube was $8 \mathrm{~cm}$ long and $3.1 \mathrm{~cm}$ diameter. The total mass of ${ }_{92}^{238} \mathrm{U}$ was 81 grams and the effective density was $1.342 \mathrm{~g} / \mathrm{cm}^{3}$. This target was also used for the N-SI-109 experiment.

The ${ }_{90}^{232} \mathrm{Th}$ target was made of a $8 \mathrm{~cm}$ long aluminum cone with an entry diameter of $2.5 \mathrm{~cm}$ and an exit diameter of $5.5 \mathrm{~cm}$. Such a geometrical shape ensured a maximum overlap with the neutron spatial distribution out of the LICORNE gas cell. 7 pellets of natural thorium were placed to achieve a total mass of $129.7 \mathrm{~g}$ with an effective density of $0.984 \mathrm{~g} / \mathrm{cm}^{3}$. This target had an intrinsic activity of $5 \mathrm{MBq}$. 

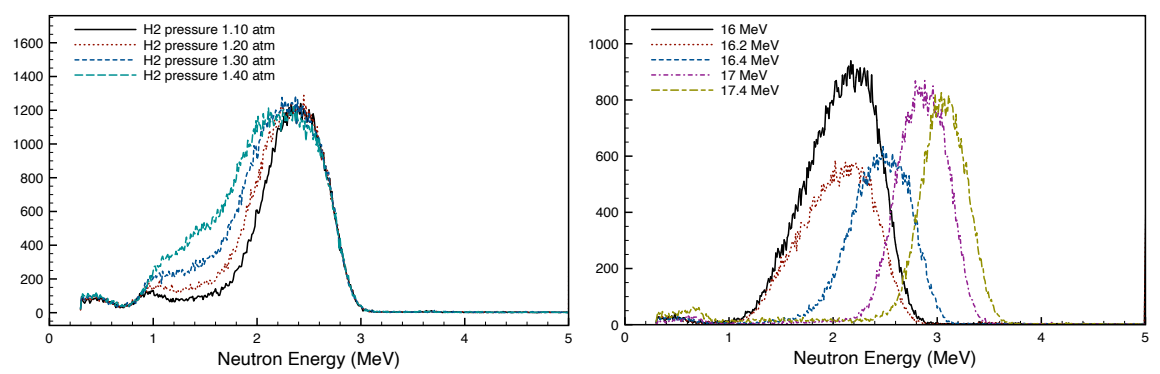

Figure 3. On the left: neutron energy spectra measured as a function of the $\mathrm{H}_{2}$ pressure in the gas cell. On the right: neutron energy spectra measured as a function of the incident lithium beam energy.

The LICORNE beam was tuned to maximize the neutron flux in the target and minimize the cone opening to avoid hitting forward detectors. Figure 3 shows how the neutron spectrum can vary with two of the LICORNE parameters: incident lithium beam energy (on the right), and $\mathrm{H}_{2}$ target density (on the left). For the two measurement a lithium beam energy of 16.75 MeV has been chosen.

\subsection{Fission trigger for the ${ }^{232} \mathrm{Th}(\mathrm{n}, \mathrm{f})$ experiment}

While the beam was on target, several processes took place:

- ${ }^{232} \mathrm{Th}$ fission, with a calculated rate of $20 \mathrm{kHz}$.

- The $\mathrm{p}\left({ }^{7} \mathrm{Li},{ }^{7} \mathrm{Li}^{\star}\right) \mathrm{p}$ reaction that produces a $478 \mathrm{keV} \gamma$-ray; An additional lead shielding has been designed to attenuate this $\gamma$ source by a factor 15 . In addition as the $\gamma$-rays are emitted in flight, Doppler shift and broadening can be observed in the $\gamma$ energy spectrum creating a structure between $470-515 \mathrm{keV}$ that prevent any coincidence with $\gamma$-rays in this energy region. The expected reaction rate is in the order of $1 \mathrm{MHz}$ with a $4 \pi$ emission.

- With an specific activity of $4.09 \mathrm{kBq} / \mathrm{g}$, this target achieved an activity of $5.2 \mathrm{MBq}$. Such an activity means a large probability of random coincidences even with the 2 ns narrow beam pulse and on a wide energy range.

After the first step of the data analysis: energy calibration, gain matching, time alignement (detailed in [12]), a first total projection of prompt $\gamma-\gamma$ matrix can be performed. The result is shown in Fig. 4. The projection of the $\gamma-\gamma$ matrix contains many $\gamma$ lines produced by $\gamma$ rays from the parasitic processes detailed earlier. To minimize contamination with unwanted $\gamma$-rays, several trigger condition have been tested. All of them rely on the fact that fission process emits a larger number of $\gamma$-rays than the parasitic ones. For instance, a trigger considering prompt multiplicity (in a time window of $50 \mathrm{~ns}$ around the beam pulse) up to 6 have been considered. If the matrices are cleaned up of unwanted $\gamma$-lines it comes with a lower statistics. To try to compensate for this loss a new approach has been considered. It relies on a multivariate analysis using neural network architecture.

\subsubsection{Neural networks}

Neural networks (NN) is an algorithm capable of correlating a list of input values to a given list of output values. This correlation is created statistically in what is named the training process. The structure is composed of $\mathrm{N}$ neurons, each of them represent a smooth step function that for an input value between 0 and 1 returns a value in the same range. The slope 


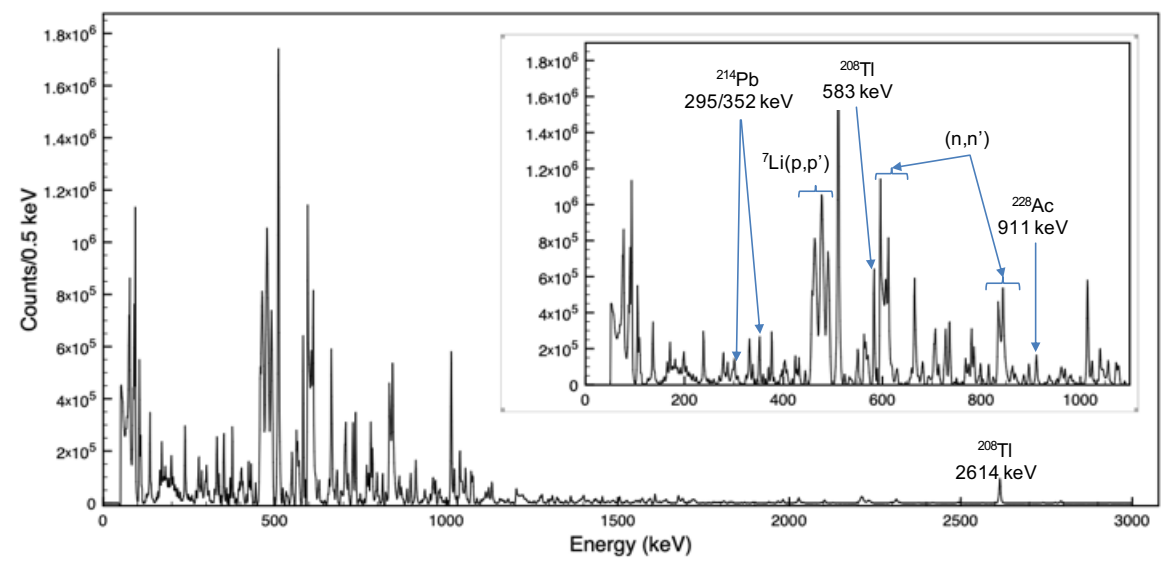

Figure 4. Total projection of a prompt-prompt $\gamma-\gamma$ matrix on the energy range 50-3000 keV. The subfigure represent a zoom on the low energy region.

of this step function and the normalisation factor at the input of the neuron is chosen during the training sequence. In other words, a neural network is capable of performing the selection of a subspace in an $\mathrm{N}$ dimension hyperspace with $\mathrm{N}$, being the number of inputs which is very similar to a graphical cut on a 2D plot.

Neural ntworks are a branch of artificial intelligence that knows a rebirth of interest in science as it is an efficient way to get the results of complicated calculations with simple algorithms. It is now commonly used for instrumentations and physics application [23-25]. The design of a neural network is made of three major steps: understanding the useful input parameters, defining the architecture, the training procedure. Then, a neural network quality is characterized by its generalization capability. That is to say to give the correct output for a new dataset.

\subsubsection{Determination of input data \& NN architecture}

The aim of the neural network is to discriminate fission from other nuclear reactions that occur at a much higher rate. The input parameters used for the NN will be measured using the $v$-Ball spectrometer and are supposed to get distinguishable values depending on the detected reaction. At the moment of its conception, the array was known to have calorimetry capabilities, high resolution energy measurement and fast timing properties. The coupling of the spectrometer with the LICORNE neutron source made the use of pulsed neutron beams possible.

As a consequence, the first pre-treatment of the data will require that all the hits are grouped in an "event" that corresponds to a $400 \mathrm{~ns}$ time window around a beam pulse. More precisely the time window starts $60 \mathrm{~ns}$ before and end $340 \mathrm{~ns}$ after a beam pulse. From this set of hits several meta-data can be calculated such as: total and average energy (prompt or delayed w.r.t. the beam pulse), multiplicity (prompt or delayed), and standard deviation of these meta-data, etc. Up to 16 variables were identified. Some NN also use non-linear combination of inputs to increase the NN predictive power. In this first approach, this possibility was not considered.

The wanted output is a decision level: a real number between 0 and 1 that corresponds to the percentage of chances to have a fission. Later, depending on the response of the neural network a decision threshold can be chosen to optimize the discrimination. 
With a defined number of inputs and outputs, the NN boundary conditions are defined: one input layer made of as many neurons as inputs and one output layer made of one neuron. In between some hidden layers need to be created. Depending on the layout of the neurons that compose the hidden layers, more or less complex linear correlations between inputs can be performed. There a unfortunately no precise prescriptions on the number of hidden layers of hidden neurons. Nonetheless, one can figure out that one hidden layer will correlate linearly all the inputs to a given output, two hidden layers will correlate quadratically all the outputs and so on. This mean that for our application two or three hidden layers consists of a reasonable maximum. The number of hidden neurons has been modified to achieve the best $\mathrm{NN}$ response after the training procedure.

\subsubsection{Training Procedure}

After a given NN layout has been chosen, all the normalisation factors and inputs weights for every neurons must be calculated. The ROOT software [17] provide a library named TMVAnalysis. This package has been created to be capable to quickly define a neural network structure and perform its training from lists of inputs taken from a ROOT file.

The next step is to build several data sets that will serve to train (fit) the neural network and to test its response. As mentioned earlier, there are three main processes that will be considered: fission, natural thorium target intrinsic activity and (p,p') reaction in the gas cell. Experimentally, several runs were acquired:

- During the commissioning of the spectrometer, an ionization chamber containing a ${ }^{252} \mathrm{Cf}$ spontaneous fission source was used. Considering the fission chamber anode signal as a "beam pulse", $400 \mathrm{~ns}$ wide events were reconstructed containing only fission. To these events, an output value of 1 was assigned.

- During the ${ }^{232} \mathrm{Th}(\mathrm{n}, \mathrm{f})$ irradiation measurement, several calibration run were performed. During these runs, the RF signal was not switched off. Again, the same $400 \mathrm{~ns}$ wide events were reconstructed containing only target intrinsic activity events. To these events, an output value of 0 was assigned.

- During the ${ }^{238} \mathrm{U}(\mathrm{n}, \mathrm{f})$ irradiation measurement, a run with the neutron beam but no target was performed. Then, the reconstructed $400 \mathrm{~ns}$ wide event contain mainly (p,p') reactions in the gas cell. To these events, an output value of 0 was assigned.

From these, two files containing meta-data of each event type and the expected output value were created. One file was used for training (named "Training Set") and the other one for checking the neural network response (named "Test Set"). In the training process, several file sizes were chosen. As the procedure is very similar to a fit, the initial naive step was to take a large number of training events. However, during the training procedure, it was shown to be not necessary.

The TMVAnalysis library training procedure consists of two steps. In the first one, the program will read all the inputs from the training set to determine normalization factors so that the input value cannot exceed 1. In a second step, it will fit all the weights of all the neurons on the test set. A user defined proportion of the test set can be used as a verification that the fit is good enough. If not, the neuron weights are reseted and the program tries a new fit.

At the end of the training procedure, the neural network is tested on a test set. The neural network response to new input data is calculated and the response is then compared with the known answer. The discrepancy between the response and the expected value serves as a parameter to evaluate a NN quality. 


\subsubsection{Neural network quality}

If the observed discrepancy characterize the quality of the neural network to a test set, it might not be easy to quantify the generalization capability, i.e. getting the correct answer for an unknown set. Thus, for a given threshold on the output the rate of true positive $v s$ false positive is a statistical information that can characterize more precisely the quality of a neural network generalization capability. It is called the receiver operating characteristic curve (or "ROC" curve) - see Fig. 5. In an ideal situation, it would be represented by a step function that takes the value 1 for any false positive rate value. In Fig. 5, continuous lines represent

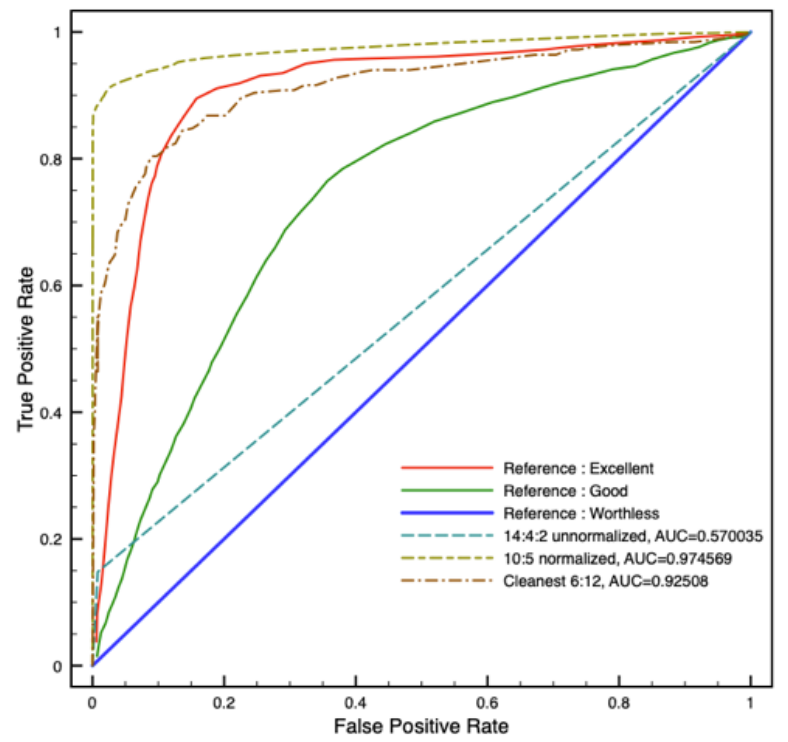

Figure 5. Receiver Operating Characteristic (ROC) curve for various neural network architecture: continuous lines represent limit cases; dashed lines represent actual network ROC curves.

prescriptions to evaluate the quality of a neural network using the ROC curve evaluator. The diagonal one represents the worst case scenario for which any decision threshold will give a $50 \%$ chance of mistake. If the NN has a ROC curve close to this one, then it is pretty much useless. The green and red curves represent a good and excellent cases, respectively. If the ROC curve is above the red one, then the neural network might be too good. In our situation, too good means that the neural network has perfectly learnt the features of the spontaneous fission of ${ }^{252} \mathrm{Cf}$ detected by a fission chamber. However it fails to generalize the features to other fissioning systems that are not detected in a fission chamber. In the domain of artificial intelligence this corresponds to overtraining. In Fig. 5, the curve labeled "10:5 normalized" is a perfect example with a success rate of $90-100 \%$ to recognize fission in the test set. When applied to the thorium data set, no fission fragment specific $\gamma$ peaks are visible in a $\gamma-\gamma$ matrix.

During the training process:

- More than fifty architectures were tested.

- Different training sets were tested. The size was changed from a few thousand events to several millions. 
- The representation of each process in the training set was also studied: equally likely, same ratio as reaction rate ratios, ...

- The expected output value was also modified to include the likeliness of processes. For example, one can assume that the (p,p') reaction is more similar to intrinsic decay in the target than to fission when comparing the input data: the expected response of the NN was 0 for both intrinsic activity and (p,p') reaction.

At the end, one neural network architecture has been selected. It is using six metadata as inputs: the prompt spatial distribution of $\gamma$-rays, the $400 \mathrm{~ns}$ average spatial distribution, the event/prompt multiplicity and the event/prompt total energy. Its ROC curve is named "Cleanest 6:12" in Fig. 5. It is close to the reference for excellent neural networks. This architecture was then used to perform the fission trigger decision in the thorium experimental data. An analysis code was written to extract from real data pulses the necessary inputs. Then, a threshold (corresponding to a confidence level of 70\%) on the NN output was set. From the selected data, a prompt-prompt (in coincidence with a beam pulse) $\gamma-\gamma$ matrix was constructed. The total projection of this matrix is presented in Fig. 6 as a red histogram. In the sub-view, the $4^{+} \rightarrow 2^{+}$and $2^{+} \rightarrow 0^{+}$transitions in ${ }^{140} \mathrm{Xe}$ are indicated. For comparison purposes, the blue histogram is a projection of a similar matrix based on a prompt multiplicity filter. The two projections have been normalized. In matrix projection resulting of a neural network based

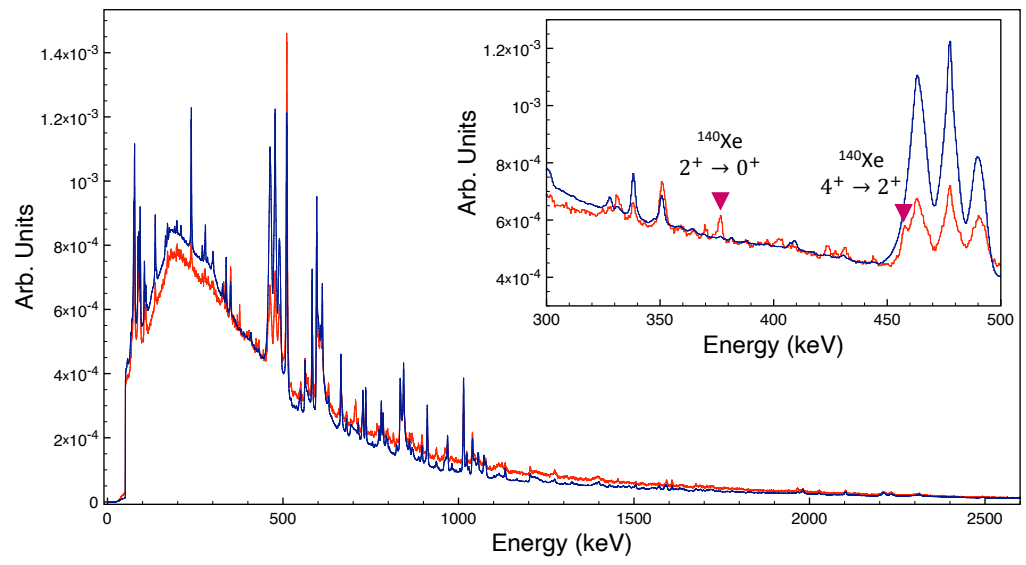

Figure 6. Total projection of two prompt-prompt $\gamma-\gamma$ matrices. The red one is obtained using a neural network based trigger. The blue one is obtained using a multiplicity filter of at least three HPGe crystal fired (after add-back and Compton suppression) in coincidence with the beam pulse. The sub-view is a zoom on the low energy region. The $4^{+} \rightarrow 2^{+}$and $2^{+} \rightarrow 0^{+}$transition of the most produced fission fragment $\left({ }^{140} \mathrm{Xe}\right)$ are shown.

decision, the main $\gamma$ peaks produced by the $\gamma$-rays emitted by the most produced fission fragment are clearly visible. It is an achievement in itself as these peaks are generally in an energy range dominated by parasitic reactions. Again, these fission fragment induced structures are not visible on the matrix projection built with a multiplicity filter. The rejection rate of the neural network algorithm seems to be reasonable as the measured fission rate (measured with the ${ }^{140} \mathrm{Xe} 2^{+} \rightarrow 0^{+}$transition) is about the same order of magnitude as the calculated one. However, this success has to be mitigated. Indeed, the measured fission rate greatly depends on the considered fission fragment and it might vary from the expected value to a fifth of the calculated rate. Added to this, construction of cubes and matrices based on the selected 
data leaves a very small amount of data, which makes the identification of weakly populated transition almost impossible. In that sense a more classical multiplicity trigger is still a robust way to address this type of data.

To conclude, the high selectivity of the NN algorithm has been demonstrated. This first try to apply that type of triggering technique highlighted the pro and the cons of such procedure. First, during experiment, no data acquisition dedicated to the construction of training set has been performed just before the actual experiment. This means that the geometry, the number of channels available, or more globally the response function of the array has changed between the training set and the experimental set we apply the neural network to. Second, the fission data set was based on a different fissioning system: ${ }^{252} \mathrm{Cf}$ instead of ${ }^{232} \mathrm{Th}$. If globally, the fission process ought to generate high $\gamma$ multiplicity events with a high total energy compare to the parasitic processes, one may argue that ${ }^{232} \mathrm{Th}$ might get closer to multiplicity and energy released by the intrinsic activity of the target [26], making the discrimination even more difficult. Last, the library used to build the neural network structure was using basic functions, a more evolved one based on python programming language [27] might provide better results.

\section{Conclusion}

In this paper, a brief overview of the $v$-Ballcampaign has been given. The $v$-Ballarray, built in a similar way to EUROBALL, EXILL, is one of the first to combine high energy resolution detectors with high time resolution crystals. During the campaign, a significant beam time has been dedicated to induce fission on ${ }^{238} \mathrm{U}$ and ${ }^{232} \mathrm{Th}$ with fast neutrons produced by the ALTO LICORNE source. The high activity of the natural thorium target, made the fission event reconstruction difficult. Thus, a new technique based on neural network has been studied. It has been proven to be successful. However, several improvements can be performed and should be implemented in the future. If given the opportunity, the construction of a real fission dataset of the fissioning system with exactly the same response function of the array is a must have. In the context of a $v$-Ball2 campaign it will have to be prepared.

\section{References}

[1] U.F.E. Collaboration, Nucl. Phys. A 520, c657 (1990)

[2] J. Eberth et al., Prog. Part. Nucl. Phys. 28, 495 (1992)

[3] F.A. Beck, Prog. Part. Nucl. Phys. 28, 443 (1992)

[4] I.Y. Lee, Nucl. Phys. A 520, c641 (1990)

[5] C. Sotty, AIP Conf. Proc. 1852, 080009 (2017)

[6] V. Werner et al., J. Phys. Conf. Ser. 366, 012048 (2012)

[7] D. Bucurescu et al., Nucl. Instr. and Meth. in Phys. Res. A 837, 1 (2016)

[8] http://gammapool.lnl.infn.it/index.htm

[9] A. Maj et al., Acta Phys. Pol. B 40, 565 (2009)

[10] M. Zieblinski et al., Act. Phys. Pol. B 44, 651 (2013)

[11] C. Ghosh et al., J. Instrum. 11, P05023 (2016)

[12] M. Lebois, N. Jovancevic, D. Thisse, R. Canavan, D. Etasse, M. Rudigier, J. Wilson, Nucl. Instrum. Methods Phys. Res. A 960, 163580 (2020)

[13] http://ipnwww.in2p3.fr/GePool/poolRules.html

[14] O.J. Roberts et al., Nucl. Instrum. Methods A 748, 91 (2014)

[15] http://www.ukndn.ac.uk 
[16] http://faster.in2p3.fr

[17] R. Brun, F. Rademakers, Nucl. Instrum. Methods Phys. Res. A 389, 81 (1997)

[18] M. Lebois et al., Acta Phys. Pol. B 50 (2019)

[19] M. Rudigier et al., Acta Phys. Pol. B 50 (2019)

[20] S. Leoni et al., Acta Phys. Pol. B 50 (2019)

[21] N. Jovancevic et al., Acta Phys. Pol. B 50 (2019)

[22] https://cc.in2p3.fr/en/

[23] E. Ronchi et al., Nucl. Instrum. Methods A 610, 534 (2009)

[24] Z. Szadkowski et al., Progress in Electromagnetics Reseach Symposium (2017)

[25] L. Gupta et al., Pattern Recog. 25, 413 (1992)

[26] J.W. Wilson et al., THEORY-5 proceeding to be published (2020)

[27] https://docs.python.org/3/reference/index.html 